\title{
Investigation of Graphene Oxide and Graphene Oxide Quantum Dots Dispersions in Organic Solvents
}

\author{
Yongqiang Yanga, Qinsheng Wang, Zheng Liu, Wenjun Liu, Yu Wen, Ling Jin, \\ Yuan Liu, Bingxian Ou, Qun Wang, Pengju Han
}

National Graphene Products Quality Supervision and Inspection Center (Jiangsu), Jiangsu Province Special Equipment Safety Supervision Inspection Institute . Branch of Wuxi, Wuxi 214174, P. R. China

ayyqyyq_2008@163.com

Keywords: graphene oxide, graphene oxide quantum dots, dispersion.

Abstract. The dispersion behavior of graphene oxide and graphene oxide quantum dots in different organic solvents has been investigated. When these dispersed solvents were placed for one year, as-prepared graphene oxide were seen to exhibit long-term stability in three solvents (ethylene glycol, $\mathrm{N}, \mathrm{N}$-dimethylformamide and $\mathrm{N}$-methyl-2-pyrrolidone) comparable to that observed for the dispersion of the same material in water. In contrast, as-prepared GOQDs can form dispersions with long-term stability in seven solvents (methanol, ethanol, 1-propanol, ethylene glycol, dimethyl sulfoxide, N, $\mathrm{N}$-dimethylformamide and $\mathrm{N}$-methyl-2-pyrrolidone). Furthermore, the dispersion ability and stability of GOQDs were obviously enhanced in other four solvents (acetone, pyridine, tetrahydrofuran and dichloromethane). The enhancing of dispersion behavior should facilitate the manipulation and processing of graphene-based materials for different applications.

\section{Introduction}

Graphene, a single atomic layer that consisted of $\mathrm{sp}^{2}$ carbon atoms covalently bonded in a hexagonal manner, has emerged in recent years as a novel and important class of materials on its own merit due to its ultrahigh surface area, excellent electrical, mechanical and thermal properties.[1,2,3] Graphene oxide (GO), as a promising route to achieve mass production of graphene, also has been studied in the context of many applications due to its special properties. [4,5] In contrast to graphene, GO has a large amount of oxygen-containing groups on the basal plane and edges, these functional groups allow the good dispersion of GO in aqueous solution. [4,5] Therefore, so far, most of the work reported on the solution-phase manipulation of GO has been carried out in aqueous media. However, the preparation of graphene oxide dispersions in other solvents, particularly organic solvents, is highly desirable because it may significantly facilitate the practical use of the materials. $[4,5,6,7,8,9,10]$

By taking the advantages of GO, several GO-water-soluble polymer composites with noticeably improved property have been obtained by simple mixing procedure in water. However, further complex chemical modification of GO with different molecules or polymers is often required for preparation of water-insoluble polymer composites, since GO cannot be readily exfoliated in most organic solvents. To date, the dispersion of graphene oxide in organic solvents has been accomplished via covalent functionalization of the graphene oxide sheets with different molecules and polymers, but the presence of such stabilizers is not desirable for most applications.[5,6,7,8] Besides, the surface-modification process usually suffers from limited scalability, time-consuming and environmental problems. Therefore, it is highly desirable to find an easy approach for the exfoliation of GO in organic solvents to simplify the fabrication procedure of graphene-polymer composites.

So far, Paredes et al. has investigated the dispersion behavior of graphene oxide in the following organic solvents: acetone, methanol, ethanol, 1-propanol, ethylene glycol, dimethyl sulfoxide (DMSO), N,N-dimethyformamide (DMF), N-methyl-2-pyrrolidone (NMP), pyridine, tetrahydrofuran (THF), dichloromethane, o-xylene, and n-hexane. It has been found that the graphene oxide dispersions exhibited long-term stability in DMF, NMP, THF, and ethylene glycol, when the size of the GO was between a few hundred nanometers and a few micrometers large, similar to the case of 
graphene oxide dispersions in water.[11] Paredes et al. suggested that the other factors besides the solvent polarity are important for determining good dispersability, but the other factors were not investigated. [11]

In our previous work, the graphene oxide quantum dots (GOQDs, the graphene sheets with lateral dimensions less than one hundred nanometer.) with uniform lateral dimensions of $\sim 50 \mathrm{~nm}$ were prepared through photo-Fenton reaction of GO.[12] Therefore, the effect of GO size was investigated for the dispersion behavior in different organic solvents in this paper.

\section{Experimental Section}

\section{Preparation of GO and GOQDs:}

GO and GOQDs were generated as described in our previous work.[12,13]

GO was prepared from graphite powder through a modified Hummers method. In a typical experiment, graphite $(2 \mathrm{~g})$ and sodium nitrate $(1 \mathrm{~g})$ were put into a $250 \mathrm{ml}$ flask at $0{ }^{\circ} \mathrm{C}$. Subsequently, concentrated $\mathrm{H}_{2} \mathrm{SO}_{4}$ was added slowly in the flask under stirring, and the temperature of mixture was kept at $5{ }^{\circ} \mathrm{C}$. The mixture was stirred continuously for 2 hours, and meanwhile $\mathrm{KMnO}_{4}$ was added in small portions to prevent the rapid temperature rise (the temperature was kept at $\sim 10{ }^{\circ} \mathrm{C}$ ). Then, the temperature of the reaction mixture was lifted to $35{ }^{\circ} \mathrm{C}$ and was stirred for 2 hours. After completion of the reaction, $90 \mathrm{ml}$ of water was poured slowly into the solution under vigorous stirring, and dark brown suspension was obtained. The suspension was treated further by adding the mixture of $\mathrm{H}_{2} \mathrm{O}_{2}$ and water. The graphite oxide was separated from the reaction mixture by filtration. The yellow-brown graphite oxide powders were washed for three times with warm diluted $\mathrm{HCl}$, and were dried finally under vacuum.

GOQDs were prepared in a photo-reactor equipped with the irradiation lamps (Bilon, Shanghai) through a photo-Fenton reaction. Briefly, $5 \mathrm{~mL}$ of $0.5 \mathrm{mg} / \mathrm{mL}$ GO aqueous suspension, $20 \mathrm{~mL}$ of 20 $\mathrm{mM} \mathrm{H} \mathrm{O}_{2}$, and $100 \mu \mathrm{L}$ of $1.0 \mathrm{mM}$ of $\mathrm{FeCl}_{3}$ were mixed in a quartz tube under vigorous stirring. The $\mathrm{pH}$ of the mixture was adjusted to 4 . The reaction was initiated by exposing the quartz tube to a mercury lamp ( $365 \mathrm{~nm}, 1000 \mathrm{~W})$. Afterward, the products were dialyzed in a dialysis bag of $3500 \mathrm{Da}$ for 3 days to remove the excess $\mathrm{H}_{2} \mathrm{O}_{2}$ and other small molecules generated during the reaction process.

\section{Dispersion of GO and GOQDs:}

For the preparation of GO and GOQDs dispersions in different solvents, the dried product was added to the solvent and sonicated in an ultrasound bath cleaner (KQ5200DB supersonic cleaner, China) for $1 \mathrm{~h}$. To allow direct comparisons between the dispersing behavior of the different solvents, a certain amount of graphite oxide powder $(\sim 5 \mathrm{mg})$ was added to a given volume of solvent $(\sim 10 \mathrm{~mL})$ in such a way that the resulting nominal concentration was adjusted to $0.5 \mathrm{mg} \mathrm{mL}-1$ for all of the solvents. Graphite oxide dispersions were tested in the following organic solvents: acetone, methanol, ethanol, 1-propanol, ethylene glycol, dimethyl sulfoxide (DMSO), N,N-dimethylformamide (DMF), N-methyl-2-pyrrolidone (NMP), pyridine, tetrahydrofuran (THF), dichloromethane, o-xylene, and n-hexane. In all of the solvents, the water content was below $0.1 \%$. Because the common solvent for the preparation of graphite oxide dispersions is water, aqueous dispersions of our as-prepared graphite oxide material were also made under exactly the same conditions as those used in the case of the organic solvents. Such water dispersions served as a reference against which the organic solvent dispersions were compared. To observe the dispersions stability of the different solvents, these solvents were placed to stand for at one year.

\section{Characterization:}

AFM (Atomic Force Microscope) images were acquired in the tapping mode with a Dimension FastScan scanning probe microscopy system (Bruker, USA). The FTIR spectra were acquired on a Nicolet IS50 FT-IR spectrometer (Thermo Fisher, America). XPS measurements were performed on an Axis Ultra DLD spectrometer (Kratos Analytical, UK) with a monochromated Al Ka source at 15 $\mathrm{kV}$. Raman spectroscopy measurement was examined by laser confocal micro-Raman spectroscopy 
using LabRam HR Evolution (Horiba Jobin Yvon, Japan) with an excitation laser beam wavelength of $473 \mathrm{~nm}$.

\section{Results and Discussion}
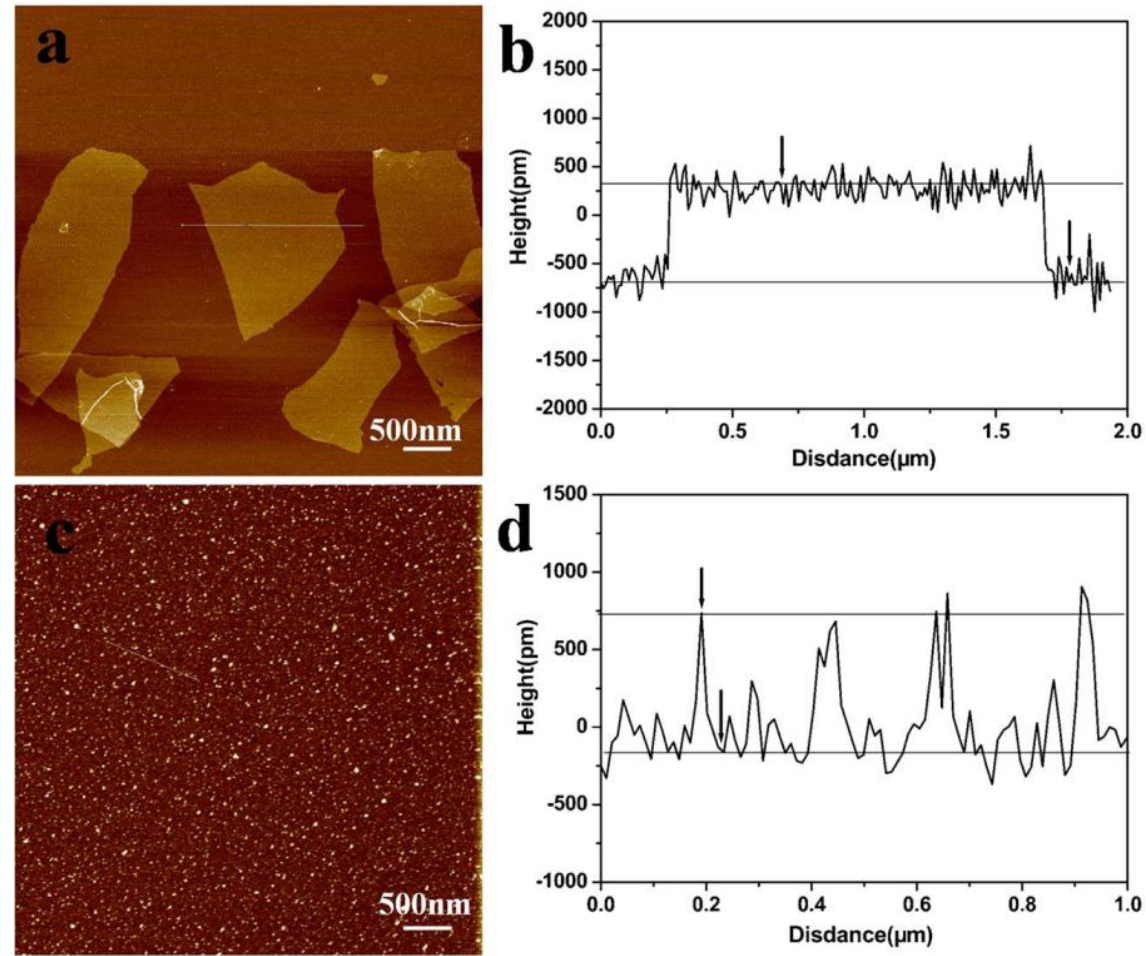

Fig. 1 Tapping mode atomic force microscopy images of individual (a) GO and (c) GQODs on mica substrate, and the height profiles of (b) GO and (d) GQODs. The corresponding thicknesses of GO and GQODs are $\sim 0.93 \mathrm{~nm}$ and $\sim 0.89 \mathrm{~nm}$, respectively.

The detailed morphology and height of as generated GO sheets and GQODs were studied by AFM, Figure 1 depicts a typical tapping mode AFM image and the crossing section profile of as-generated GO sheets and GQODs deposited on freshly cleaved mica surface. The uniform contrast of the AFM images of GO sheets (Fig. 1a) implies that all of them possess the same thickness. The thickness is about $0.9 \mathrm{~nm}$ measured from the crossing section profile curve (Fig. 1b). All GQODs with an average lateral size of $\sim 50 \mathrm{~nm}$, and thickness of $\sim 0.9 \mathrm{~nm}$. Such thickness is significantly larger than that of single-layer pristine graphene $(\sim 0.34 \mathrm{~nm})$ and is generally attributed to the presence of oxygen-containing functional groups attached on both sides of the graphene sheet and to the atomic scale roughness arising from structural defects ( $\mathrm{sp}^{3}$ bonding) generated on the originally atomically flat graphene sheet. [14] Thus, individual graphene oxide sheets are expected to be thicker $(\sim 1.0 \mathrm{~nm})$ than individual pristine graphene sheets $(\sim 0.34 \mathrm{~nm})$.

Figure 2 shows the typical FTIR spectra of GO and GQODs. The intensities of the FTIR peaks corresponding to the oxygen functionalities, such as the $\mathrm{C}=\mathrm{O}$ stretching vibration peak at $\sim 1710 \mathrm{~cm}^{-1}$, the vibration and deformation peaks of $\mathrm{O}-\mathrm{H}$ groups at $\sim 3200 \mathrm{~cm}^{-1}$ and $\sim 1380 \mathrm{~cm}^{-1}$, respectively, the $\mathrm{C}-\mathrm{O}$ (epoxy) stretching vibration peak at $\sim 1220 \mathrm{~cm}^{-1}$, and the $\mathrm{C}-\mathrm{O}$ (alkoxy) stretching peak at $\sim 1040$ $\mathrm{cm}^{-1} \cdot[5,13]$ These observations confirmed that most oxygen functionalities in the GO and GQODs.

Figure 3 is the Raman spectra of GO and GQODs. The relative intensities of G band at $\sim 1600$ $\mathrm{cm}^{-1}$ corresponding to the first-order scattering of the $E_{2 \mathrm{~g}}$ mode, and $\mathrm{D}$ band at $\sim 1357 \mathrm{~cm}^{-1}$, arising from a breathing mode of k-point phonons of $A_{1 g}$ symmetry.[12] The ratio of $D$ to $G$ band $\left(\mathrm{I}_{\mathrm{D}} / \mathrm{I}_{\mathrm{G}}\right)$ of as-generated GQODs via photo-Fenton reaction is stronger than that of the GO. These results further confirm that the GQODs generated through photo-Fenton reaction of GO have a defect-free basal plane, and lead us to believe the photo-Fenton reaction takes place at the sites of the carbon atoms connected with the oxygen containing group, but not at the areas with uniform pristine graphene structures.[12] 


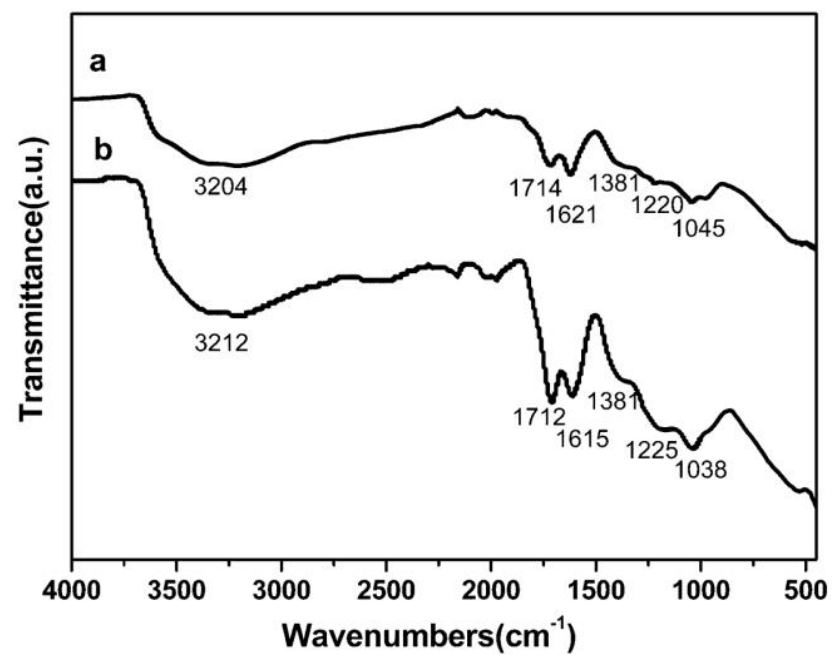

Fig. 2 FTIR spectra of (a) GO and (b) GQODs.

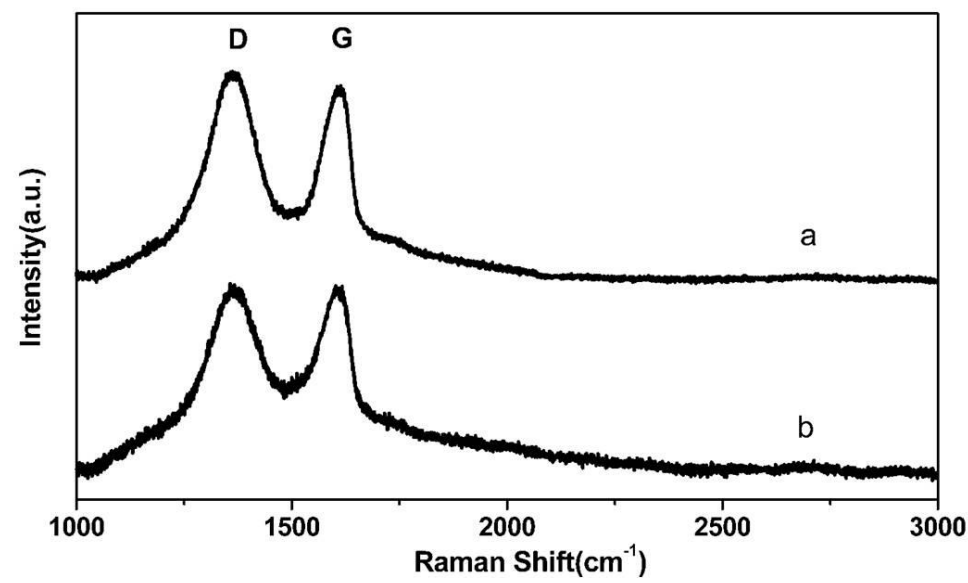

Fig. 3 Raman spectra of raw GO (a) and as-generated GQODs (b).

XPS measurements were performed to quantitative analysis of the oxygen-containing functional groups. The XPS band of the $\mathrm{C} 1 \mathrm{~s}$ core level was deconvoluted into four components (Fig. 4), corresponding to $\mathrm{C}=\mathrm{C} / \mathrm{C}-\mathrm{C}$ in aromatic rings, $\mathrm{C}-\mathrm{O}$ (epoxy and alkoxy), $\mathrm{C}=\mathrm{O}$ (carbonyl), and $\mathrm{COOH}$ (carboxylic) groups, respectively, which were in good agreement with the FTIR result (Fig. 2).[12,13]
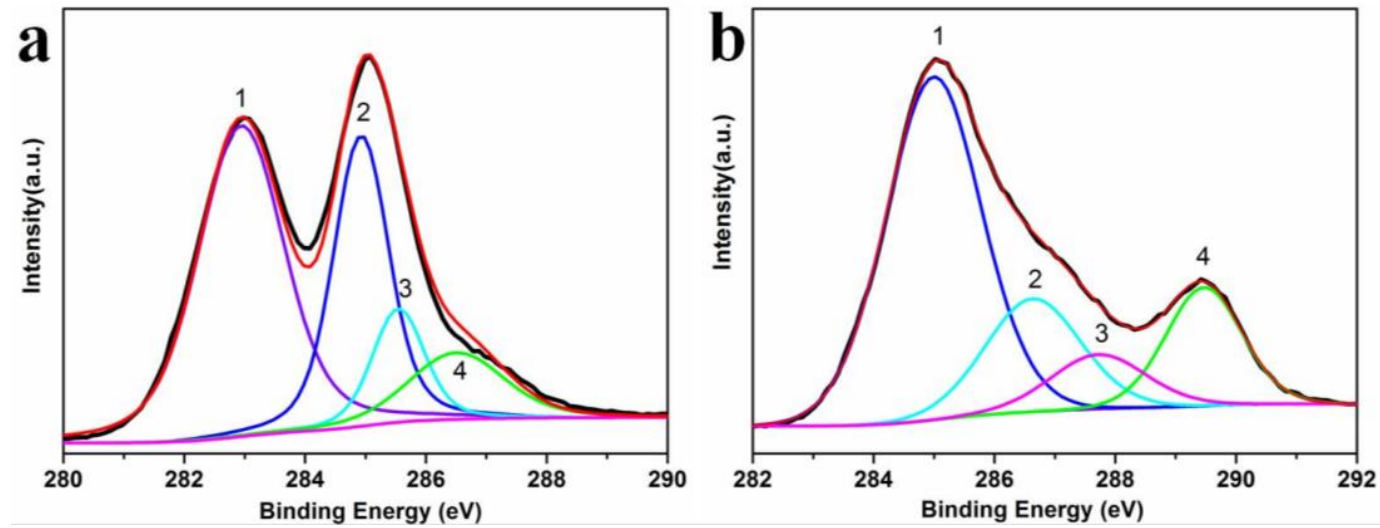

Fig. 4 XPS spectra of C1s of GO (a) and GOQDs (b). The peaks 1, 2, 3and 4 correspond to $\mathrm{C}=\mathrm{C} / \mathrm{C}-\mathrm{C}$ in aromatic rings, $\mathrm{C}-\mathrm{O}$ (epoxy and alkoxy), $\mathrm{C}=\mathrm{O}$, and $\mathrm{COOH}$ groups, respectively.

As mentioned in the Experimental Section, the as-prepared GO and GOQDs material were dispersed in 13 organic solvents and water to a nominal concentration of $0.5 \mathrm{mg} \mathrm{mL}^{-1}$ with the aid of bath ultrasonication, and the dispersions were then allowed to settle for one year. Figure 5 shows digital pictures of all of the dispersions immediately after sonication (top) and one year later sonication (bottom). For the just sonicated samples, it can be noticed that GO could be dispersed in almost all of the solvents, except dichloromethane, n-hexane, and, to a lesser extent, acetone, 
methanol and o-xylene. In contrast, GOQDs could also be dispersed in almost all of the solvents, except n-hexane, and, to a lesser extent, acetone and o-xylene. Especially a special one is GOQDs dispersed in upper portion of dichloromethane with stratification phenomenon.

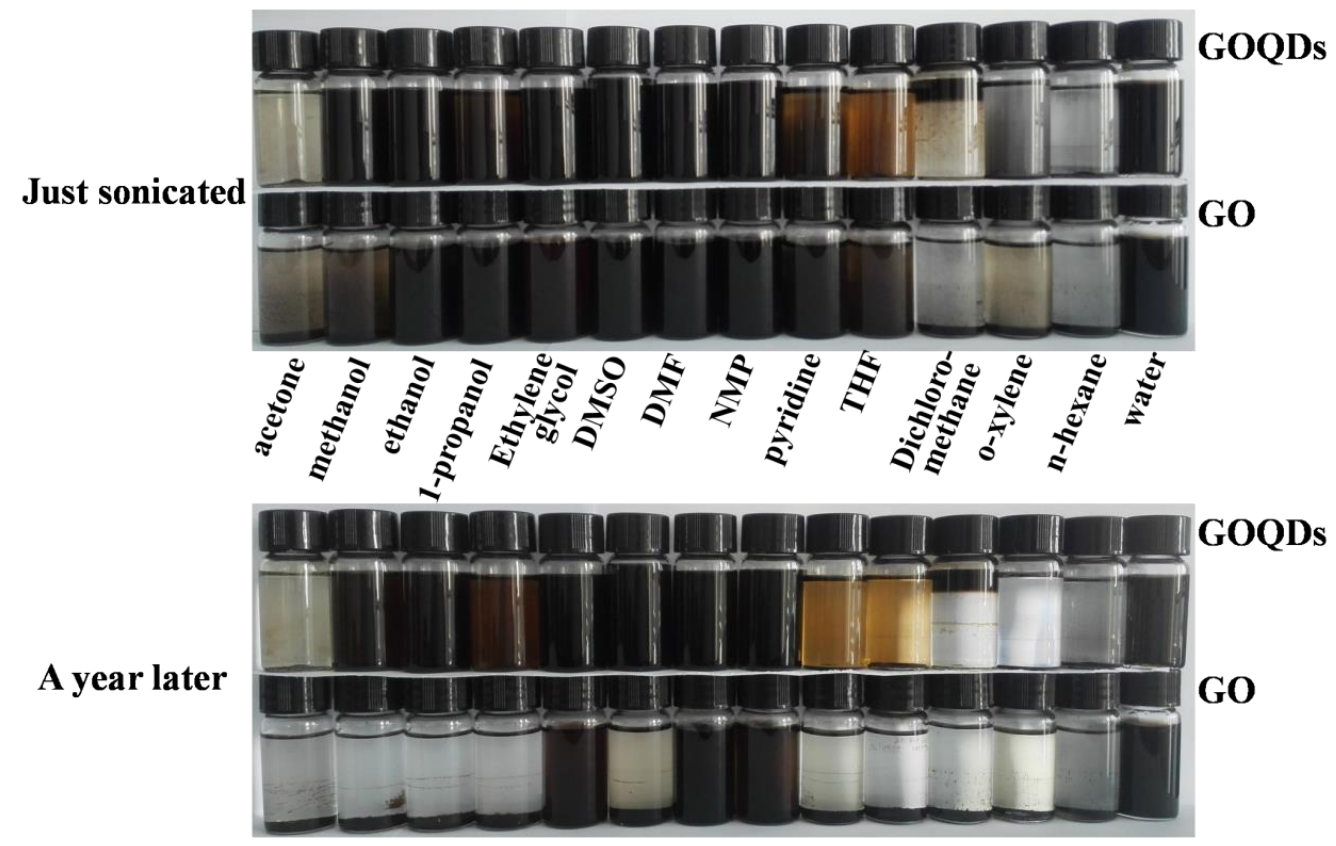

Fig. 5 Digital pictures of as-prepared GO and GOQDs dispersed in water and 13 organic solvents through bath ultrasonication (1h). Top: dispersions immediately after sonication. Bottom: dispersions 1 year after sonication.

To observe the dispersions stability of the different solvents, these solvents were placed to stand for at one year. In fact, many of these dispersions displayed only short-term stability and precipitated completely in a matter of hours to a few days, when the GO were dispersed in the different solvents. This was the case for acetone, methanol, ethanol, 1-propanol, pyridine, THF, dichloromethane, $\mathrm{o}$-xylene and n-hexane. However, the same cases were only happened for dichloromethane and o-xylene, when the GOQDs were dispersed in the different solvents. Even one year later, GO still could exhibit long-term dispersion stability in water and the four mentioned organic solvents (ethylene glycol, DMF and NMP) were seen to exhibit long-term stability comparable to that observed for the dispersion of the same material in water. And a small amount of GO also dispersed in DMSO. By contrast, under identical preparation conditions, as-prepared GOQDs also could exhibit long-term dispersion stability in water and the seven mentioned organic solvents (methanol, ethanol, 1-propanol, ethylene glycol, DMSO, DMF and NMP). Besides, the dispersion ability and stability of GOQDs were obviously enhanced in acetone, pyridine and THF. And the GOQDs which dispersed in upper portion of dichloromethane with stratification phenomenon were still stability.

Previous research by Paredes et al. has suggested that as-prepared graphene oxide could stable dispersed in some solvents may due to different polar of the solvent molecules, which is still not sufficient, and the point will necessitate further in-depth investigations.[11] In this paper, the result showed the GO and GOQDs have similar surface properties (see FTIR and XPS spectroscopy results) except the size of materials, though the long-term dispersion stability of GOQDs in some solvents were obvious enhanced than that of GO. The result indicated the size of GO and GOQDs is also one important effect for the dispersion stability of graphene oxide materials in the solvents, besides the polar of the solvent molecules.

\section{Conclusions}

We have identified several organic solvents (methanol, ethanol, 1-propanol, ethylene glycol, DMSO, DMF and NMP) in which as-prepared GOQDs with uniform lateral dimensions of $\sim 50 \mathrm{~nm}$ can form dispersions with long-term stability. Furthermore, the dispersion ability and stability of GOQDs were 
obviously enhanced in acetone, pyridine and THF. And the GOQDs which dispersed in upper portion of dichloromethane with stratification phenomenon were still stability. In contrast, under identical conditions, the GO with lateral dimensions of several micrometers can dispersed long-term stability in the four mentioned organic solvents (ethylene glycol, DMF and NMP). The result indicated reducing of the GO size can also enhanced the dispersion stability of graphene oxide materials in the solvents, besides the polar of the solvent molecules. The result is currently used for the preparation of many graphene-based materials and therefore should facilitate the further manipulation and processing of these materials.

\section{Acknowledgements}

This work was financially supported by the Science Foundation of Jiangsu Province Special Equipment Safety Supervision Inspection Institute (KJ(Y) 2015012).

\section{References}

[1] Novoselov, K. S.; Geim, A. K.; Morozov, S. V.; Jiang, D.; Zhang, Y.; Dubonos, S. V.; Grigorieva, I. V.; Firsov, A. A. Science 2004, 306, 666-669

[2] Loh, K. P.; Bao, Q.; Ang, P. K.; Yang, J. J. Mate. Chem. 2010, 20, 2277-2289.

[3] Rao, C. N. R.; Sood, A. K.; Subrahmanyam, K. S.; Govindaraj. A. Angew. Chem. Int. Ed. 2009, $48,7752-7777$.

[4] Zhu, Y.; Murali. S.; Cai, W.; Li, X.; Suk, J. W.; Potts, J. R.; Ruoff, R. S. Adv. Mater. 2010, 22, 3906-3924.

[5] Dreyer, D. R.; Park, S.; Bielawski. C. W.; Ruoff, R. S. Chem. Soc. Rev. 2010, 39, 228-240.

[6] Huang, X.; Qi, X.; Boey, F.; Zhang, H. Chem. Soc. Rev. 2012, 41. 666-686.

[7] Wang, Y.; Shi, Z.; Fang, J.; Xu, H.; Yin, J. Carbon, 2011, 49, 1199-1207.

[8] Wang, Y.; Shi, Z.; Yin, J. Polymer, 2011, 52, 3661-3670.

[9] Cai, D.; Song, M.; Xu, C. Adv. Mater. 2008, 20, 1706-1709.

[10] Cai, D.; Song, M. J. Mater. Chem. 2007, 17, 3678-3680.

[11] Paredes, J. I.; Villar-Rodil, S.; Martinez-Alonso, A.; Tascon, J. M. D. Langmuir 2008, 24, 10560-10564.

[12] Zhou, X.; Zhang, Y.; Wang, C.; Wu, X.; Yang, Y.; Zheng, B.; Wu, H.; Guo, S.; Zhang, J. ACSNANO 2012, 8, 6592-6599.

[13] Zhang, J.; Yang, H.; Shen, G.; Cheng, P.; Zhang, J.; Guo, S. Chem. Commun. 2010, 46, 1112-1114.

[14] Park, S.; Ruoff, R. S.; Nat. Nanotechnol. 2009, 4, 217-224. 